\title{
¿Inteligencia de la pasión? En búsqueda de una educación contemporánea integral e inteligentemente apasionada
}

\author{
Passion Intelligence? In search of a comprehensive and intelligently \\ passionate contemporary education
}

\begin{abstract}
Inteligência de paixão? Em busca de uma educação contemporânea abrangente e inteligente e apaixonada
\end{abstract}

Cynthia Lizeth Ramos Monsivais Universidad Autónoma de Tamaulipas, Facultad de Comercio, Administración y Ciencias Sociales, México cynthia.ramos@docentes.uat.edu.mx https://orcid.org/0000-0002-4852-4482

\section{Resumen}

En el presente trabajo se introduce el concepto inteligencia de la pasión orientada al logro de objetivos, por lo que se aborda la necesidad de desarrollarla para lograr una educación humana y significativa. En tal sentido, se concibe la pasión como una herramienta educativa que permite motivar al alumnado en la atención, elemento vital para conseguir una educación integral. Asimismo, se describe el origen filosófico desde la ilustración de los términos emoción y pasión, para lo cual se consideró el pensamiento de Kant, Descartes, Goleman, Gardner y Csikszentmihalyi para contextualizar su importancia en el marco teórico educativo de la neurociencia, la inteligencia emocional y las inteligencias múltiples. Por otra parte, se destaca la vinculación de ciencias como la psicología, la filosofía y la educación para comprender de manera holística la esencia del ser, y se propone incluir la inteligencia de la pasión en los programas educativos con la intención de formar jóvenes apasionados por el trabajo que sean productivos, creativos, con iniciativa y convicción, y que busquen lograr y cumplir sus metas y sentir gozo durante el proceso. 


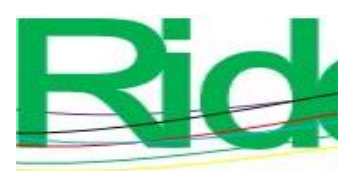

Revista Iberoamericana para la Investigación y el Desarrollo Educativo ISSN $2007-7467$

Palabras clave: educación integral, inteligencia de la pasión, meditación, motivación, neurociencia.

\begin{abstract}
The present work addresses the need to develop the passion intelligence oriented to the achievement of objectives to achieve a humane and meaningful education. Passion is conceived as an educational tool that allows students to be motivated in attention, achieving a comprehensive education as the final goal. The philosophical origin is described from the illustration of the terms "emotion" and "passion" considering the thought of Kant, Descartes, Goleman, Gardner and Csikszentmihalyi to contextualize its importance in the educational theoretical framework of neuroscience, emotional intelligence, and multiple intelligences.

The linking of sciences such as psychology, philosophy and education was highlighted to holistically understand the essence of being and it is proposed to include the intelligence of passion in educational programs with the intention to train young people to be passionate about work, productive, creative, with initiative and conviction, who seek to achieve and fulfill their goals and feel joy during the process.
\end{abstract}

Keywords: comprehensive education, passion intelligence, meditation, motivation, neuroscience.

\title{
Resumo
}

No presente trabalho é introduzido o conceito de inteligência da paixão orientada para a realização de objetivos, razão pela qual se aborda a necessidade de desenvolvê-la para uma formação humana e significativa. Nesse sentido, a paixão é concebida como uma ferramenta educativa que permite aos alunos serem motivados no cuidado, elemento vital para o alcance de uma formação integral. Da mesma forma, a origem filosófica é descrita a partir da ilustração dos termos emoção e paixão, para os quais o pensamento de Kant, Descartes, Goleman, Gardner e Csikszentmihalyi foi considerado para contextualizar sua importância no quadro teórico educacional da neurociência, inteligência emocional e inteligências múltiplas . Por outro lado, destaca-se a articulação de ciências como psicologia, filosofia e educação para compreender holisticamente a essência do ser, e se propõe incluir a inteligência da paixão nos programas educacionais com o intuito de formar jovens 


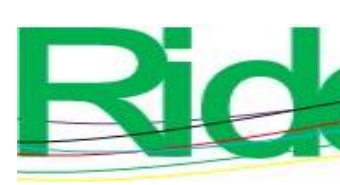

Revista Iberoamericana para la Investigación y el Desarrollo Educativo ISSN $2007-7467$

apaixonados pelo trabalho que são produtivos, criativos, com iniciativa e convicção, e que buscam atingir e cumprir seus objetivos e sentir alegria durante o processo.

Palavras-chave: educação abrangente, inteligência da paixão, meditação, motivação, neurociência.

Fecha Recepción: Diciembre 2020

Fecha Aceptación: Mayo 2021

\section{Introducción}

La Secretaría de Educación de Tamaulipas (SET) (2017) concibe a la educación como "un factor esencial para el desarrollo de las naciones. Es una herramienta de progreso y motor de cambio asociado a la movilidad social” (p. 14), de ahí que sea valorada como un medio "para generar paz, restablecer el orden y el estado de derecho, sin combatir con más violencia la inseguridad y la delincuencia” (p. 10). De acuerdo con Gardner (2012), el objetivo de la educación es formar personas, "transmitir valores, modelar roles, dominar notaciones y disciplinas" (p. 34), mientras que para García, Álvarez, González y Guzmán (2019) "formar a un estudiante implica la responsabilidad de formar a un nuevo ser humano apto, eficiente y bien preparado para un nuevo futuro" (p. 4).

Desde otra perspectiva, la visión fundamental de la educación también se relaciona “exponer lo verdadero, lo bello y lo bueno", donde "lo verdadero pertenece al ámbito de la verdad y a su contraparte: lo que es falso e indeterminable. Lo bello, al ámbito de la belleza, que incluye también a lo que carece de esta como las experiencias y objetos desagradables. Finalmente, en el ámbito de la moral se encuentra lo que se juzga como bueno y malo" (Gardner, 2012, p. 16). En esa línea, Burlando (2011) afirma que para Descartes la moral es "hacer lo que se juzga mejor" (p. 12), y que "el bien supremo de cada persona solo consiste en una voluntad firme para hacer el bien y el contento que esto produce" (p. 4).

Tomando como fundamento ideales vinculados con los antes señalados, diversas universidades han incorporado en sus lemas palabras que se refieren a esos principios. Una muestra de ello es el lema "Verdad, belleza, probidad" de la Universidad Autónoma de Tamaulipas (UAT), donde verdad hace referencia a la descripción de la conformidad de las cosas con su representación mental, belleza se asocia con la propiedad de las cosas que hace amarlas, infundiendo el deleite espiritual, y probidad se vincula con la honradez y la rectitud de ánimo e integridad en el obrar (UAT, 2021). 

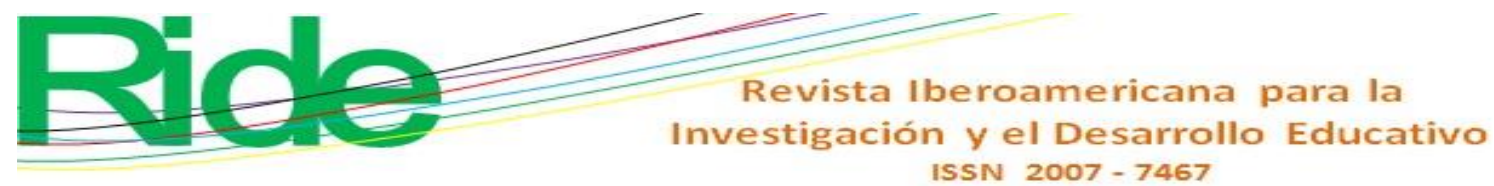

Ahora bien, a partir del siglo XVI surgieron dos concepciones sobre el modo de llegar al conocimiento: el empirismo y el racionalismo (Pimienta, De la Orden y Estrada, 2018). El empirismo es representado por Francis Bacon y John Locke, y el racionalismo por René Descartes. El empirismo "considera a la experiencia como base del saber científico", mientras que el racionalismo "enfatiza el papel del pensamiento y de la razón como fundamento de todo conocimiento" (Pimienta et al., 2018, p. 7).

A medida que la sociedad fue progresando y se comenzaron a enriquecer y a diferenciar los tipos de conocimiento, surgieron diversas ciencias que se "independizaron del tronco único y común: la filosofia" (Ramírez, 2010, p. 8). Las ciencias se dividieron en formales y fácticas, donde "las formales abarcan las ciencias exactas como la lógica y las matemáticas y las fácticas a las naturales y las sociales" (Pimienta et al., 2018, p. 8). Luego, como afirma Ferreiro (2012), en el siglo XX, con el objetivo de comprender el proceso de enseñanza-aprendizaje, "surgen diversos paradigmas psicopedagógicos como el conductismo, humanismo, cognitivo, sociocultural y constructivista" (p. 8), así como uno más reciente denominado conectivista, el cual pretende entender el aprendizaje en la era digital (Sánchez, Costa, Mañoso, Novillo y Pericacho, 2019). Este ha surgido debido a una sociedad moderna que presenta un déficit atencional derivado de las constantes distracciones y tecnologías emergentes. Esta afirmación se sustenta en estudios que demuestran que en 47 $\%$ del tiempo las personas no están prestando atención a lo que supuestamente deberían estar atendiendo (Raij, 2019).

Por ello, es lógico que se requieran nuevas estrategias que permitan comprender los procesos cognitivos del aprendizaje y adoptarlos para mejorar la educación actual. En concordancia con esta idea, y para lograr una formación integral, se resalta la importancia de impulsar la interdisciplinariedad entre ciencias como la psicología, la filosofía y la educación. Así, de acuerdo con Jiménez, Mendoza, Aimaretti, Flores, Montalvo y Mena (2020), surge "el movimiento de la mente, el cerebro y la educación conocido como neuroeducación que cada vez gana más impulso en la investigación científica" (p. 12). Al respecto, Navarro (2020) añade que la neurociencia se debe estudiar de manera integral y complementaria para entender cómo el cerebro interpreta el entorno, las relaciones interpersonales, la colaboración laboral, el comportamiento humano o el aprendizaje. 


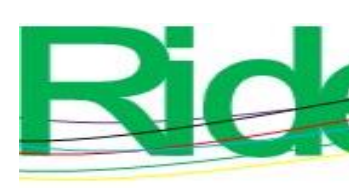

Revista Iberoamericana para la
Investigación y el Desarrollo Educativo
ISSN $2007-7467$

Lázaro (2012) argumenta que la utilidad de las pasiones radica en que ayudan a que el alma pueda retener las percepciones que le convienen, y "sin ellas el alma las podría borrar con facilidad" (p. 8). Además, señala que el objetivo del hombre es lograr que la pasión "no estropee, ni impida el autodominio, la autodeterminación y la autoestima” (p. 9).

Kant marca una diferencia entre las emociones, las pasiones y los sentimientos. Para él, la emoción es "la dimensión orgánica de un sentimiento, sin ningún tipo de acción”. En cambio, “el sentimiento es más amplio, algo del espíritu, el aspecto subjetivo de la sensación que puede producir placer o dolor, y la pasión es la facultad de desear, incorporando contenido intencional con connotaciones moralmente negativas" (pp. 2-3). Para Kant las pasiones y las emociones son negativas porque bloquean la razón y la actividad reflexiva: la emoción de manera momentánea y la pasión de manera permanente. "En la emoción, la libertad del espíritu queda suspendida, pero en la pasión es anulada" (González, 2015, p.15). En ese sentido, Urbina y Ávila (2013) afirman que "las pasiones permanecen, no son fugaces como las emociones y sobreviven gracias al sustrato racional que las caracteriza” (p. 12).

Kant explica que la razón no puede vencer a las pasiones. Según González (2015), son inclinaciones que "subordinan la actividad reflexiva a sus propios fines irracionales y contaminan desde raíz el obrar racional", de manera que privan al humano de libertad. Expone como ejemplo lo siguiente: "La indignación como cólera es una emoción: pero como odio (deseo de venganza) es una pasión” (p. 15). En contraste, Urbina y Ávila (2013) argumentan que "la pasión no es una fuerza que debamos desterrar de nuestras vidas" porque “dispone a la persona a la acción para que las intenciones de la razón no queden solo en añoranzas". Añade que "esto es posible en la medida en que se cargue de energía física la actividad cerebral, para orientar estos impulsos al logro de los propósitos” (p. 7).

Goleman, Kaufman y Ray (2016) definen la pasión como "el impulso de hacer algo por el mero placer de hacerlo" y afirman que "el término psicológico para esto es motivación intrínseca" (p. 37). Teresa Amabile incluye la pasión como parte de "los tres componentes de la creatividad: pericia, habilidades de pensamiento creativo y pasión" (citado por Goleman, Kaufman y Ray, 2016, pp. 35-37). Por otra parte, Nelson (2019) incluye a la pasión en la lista de las emociones positivas, al igual que la gratitud, confianza, satisfacción, empatía, curiosidad, felicidad, esperanza, humildad, aceptación, generosidad, benevolencia, amor e interés. Sostiene que "sentir emociones positivas sobre lo que quieres, ayuda literalmente a lograr los sueños” (p. 288). Navarro (2020) considera que los sueños se 


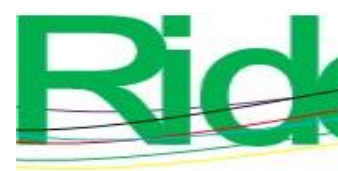
Revista Iberoamericana para la
Investigación y el Desarrollo Educativo
ISSN $2007-7467$

vuelven reales durante el proceso creativo y define la creatividad como "la capacidad de innovar, de aportar nuevas ideas, tecnologías o expresiones artísticas” (p. 6).

\section{La motivación y el logro de objetivos}

Lograr objetivos es importante para toda persona que busca la autorrealización. Existen aptitudes emocionales vinculadas al logro de objetivos, como el afán de triunfo y la iniciativa. Estas aptitudes son parte de una de las cinco dimensiones (motivación) que componen la inteligencia emocional en el modelo de Goleman. Según la Real Academia Española, la motivación es "el conjunto de factores internos y externos que motivan y determinan las acciones de una persona" (citada por Márquez, Azofeita y Rodríguez, 2019, p. 2).

Gardner (2012) afirma que cuando la motivación es intrínseca y surge por el propio placer de saciar la curiosidad, hace que la persona sea más perseverante y entre al estado de flujo. El estado de flujo requiere que la atención, la motivación y la situación se encuentren y equilibren para lograr armonía y plenitud (Raij, 2019), y se relaciona con la felicidad, el sentido de vida y la motivación intrínseca (Csikszentmihalyi, 2000). En el estado de flujo las personas están tan concentradas en una actividad que "pierden la noción del tiempo, del espacio, de las preocupaciones y del dolor" (Gardner, 2012, p. 88). En pocas palabras, "la experiencia es tan placentera que las personas la realizan incluso aunque tenga un gran costo" (Csikszentmihalyi, 2000, p. 14).

Goleman (2015) opina que las personas que se encuentran en este estado se sienten felices y obtienen mejores resultados debido a que trabajan al máximo sus capacidades. En ese sentido, describe tres modos para entrar en el estado de flujo: el primero "consiste en adecuar la tarea al conjunto de destrezas de la persona, el segundo, en encontrar un trabajo que le apasione y el tercero a través del desarrollo de una concentración total” (p. 133). Añade que muchos de los empresarios exitosos afirman que lo que los mueve es "la pasión de guiar, de servir a sus clientes, de apoyar a una causa o un producto" (p. 111). Por ello, la frase "sigue tu pasión y no tendrás que volver a trabajar” jamás tuvo tanto sentido.

La psicología positiva también puede ayudar al logro de objetivos a través del enfoque coaching. Según Cantón, Sánchez y Peris (2019), el coaching funciona como una estrategia que permite "modificar la conducta través de la reflexión, el autodescubrimiento, el aprendizaje y la acción; en donde el experto genera un entorno que facilita que el cliente 

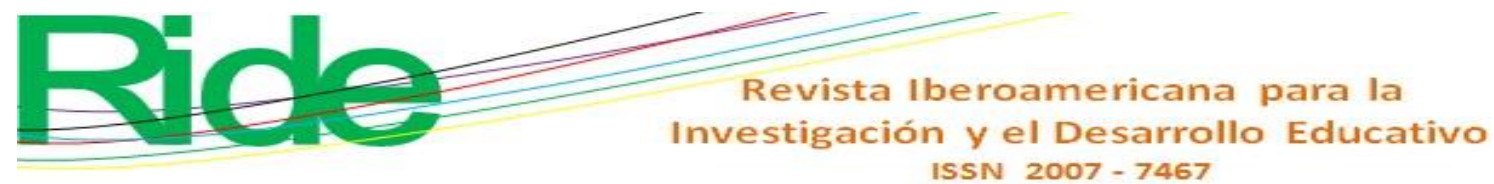

logre cumplir sus objetivos utilizando sus propios recursos" (p. 3). Para estos autores el desarrollo de estrategias terapéuticas para mejorar la experiencia emocional positiva y prevenir psicopatologías originadas por emociones negativas es uno de los objetivos de la psicología positiva. "La psicología positiva se vincula con el bienestar psicológico a través de sus dos enfoques: bienestar hedónico, vinculado a la felicidad y el bienestar eudaimónico relacionado con el desarrollo personal, propósito de vida y autorrealización" (p. 3).

\section{Comprendiendo la esencia humana: el origen de la pasión}

En el siglo XVIII, el filósofo René Descartes comenzó el estudio de las pasiones para entender "qué son, dónde, cómo y por qué se producen y para qué sirven” (citado por Lázaro, 2012, p. 3). Para comprender el origen de las pasiones es importante conocer las dualidades que integran al ser humano. Para Platón el humano es una dualidad entre el alma y cuerpo; para Marcel la dualidad es ser-tener, y para Descartes sujeto-objeto (Selles, 2007).

En el intento de explicar la complejidad humana, Selles (2007) invita a ver al humano como un compuesto integrado por cuatro dimensiones: naturaleza, esencia, instrumentos nativos de la persona y el acto de ser personal. La naturaleza corresponde a la vida recibida que es heredada por los padres. La esencia refiere a la vida añadida y corresponde a lo que cada persona suma a su vida en forma de conocimiento: hábitos cognoscitivos que cultivan la inteligencia y las virtudes adquiridas que enriquecen a la voluntad. Los instrumentos nativos son tres capacidades con las que se nace y que también se pueden desarrollar: la capacidad de juzgar de manera correcta, lo que permite conocer y gobernar la esencia y naturaleza humana; la capacidad para comprender la existencia de la realidad externa; y la sabiduría que permite a la persona conocerse a sí misma. Finalmente, "el espíritu se refiere al lenguaje, al acto de ser, al corazón humano que en conjunto se le puede llamar vida personal" (p. 4).

Por su parte, Descartes separa al sujeto del objeto con una esfera propia para cada uno: "La filosofía y la investigación reflexiva compuesta por sujetos que se plantean problemas de existencia, conciencia y comunicación; y la ciencia e investigación objetiva que refiere a los objetos que son sometidos a observación, experimentos y manipulaciones" (citado por Trujillo, 2007, p. 5). Esta dualidad se puede observar de la siguiente manera: alma-cuerpo, espíritu-materia, calidad-cantidad, finalidad-causalidad, sentimientos-razón, libertad-determinismo y existencia-esencia. 


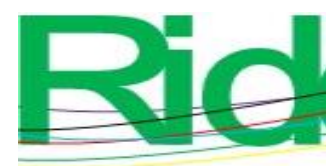

Revista Iberoamericana para la
Investigación y el Desarrollo Educativo
ISSN $2007-7467$

neurocognitivo de la persona humana y aspira a estudiar los procesos neurocognitivos de aprendizaje y de enseñanza en el aula" (p. 13).

En esa línea, con el objetivo de formar neurocientíficos, la Universidad Autónoma Nacional de México (UNAM) desde 2016 oferta la única licenciatura en Neurociencias en América Latina a través de la Facultad de Medicina en colaboración con el Instituto de Fisiología Celular, el Instituto de Neurobiología, la Facultad de Ciencias y la Facultad de Psicología (García et al., 2019).

\section{Los diferentes tipos de inteligencias y sus relaciones}

La inteligencia — según Selles (2007) — es una facultad humana relacionada con el pensamiento, la razón y el entendimiento. Para Gardner (1999, citado por Mora y Martin, 2007) la inteligencia es la "capacidad humana intelectual útil e importante para resolver problemas y crear productos de valor" (p. 5) En ese sentido, expone la teoría de las inteligencias múltiples en donde se dan a conocer ocho diferentes tipos de inteligencia que son útiles en un ambiente determinado.

La teoría de las inteligencias múltiples sugiere que la persona puede estar más motivada para aprender cuando tiene talento en la actividad que realiza. "La inteligencia es la base de la creatividad y la persona será más creativa en los campos en que posee mayores fortalezas" (Goleman et al., 2016, p. 94). La inteligencia emocional propuesta por Goleman (1995) se refiere a la capacidad de reconocer y orientar las emociones propias y ajenas para el logro de objetivos. La inteligencia emocional se integra de cinco dimensiones y 25 aptitudes que se muestran en la figura 1. 

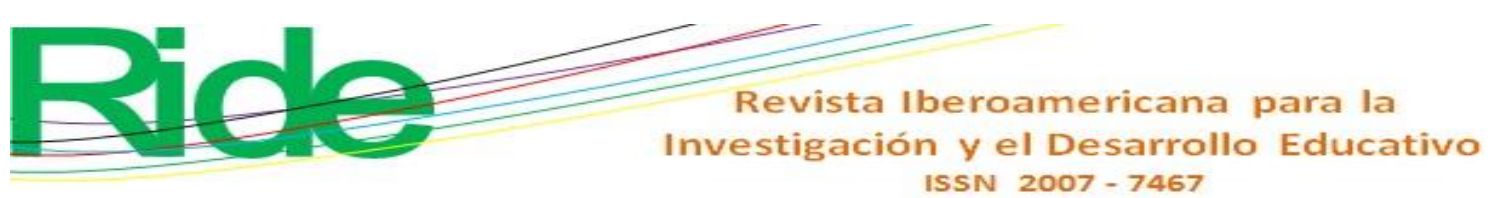

Figura 2. Relación entre las aptitudes de inteligencia emocional e inteligencia de la pasión

\begin{tabular}{|c|c|}
\hline Aptitudes personales & Aptitudes sociales \\
\hline Autoconocimiento & Empatía \\
\hline - Conciencia emocional & - Comprender a los demás \\
\hline - Autoevaluación precisa & - Ayudar a los demás a desarrollarse \\
\hline - Confianza en uno mismo & - Aprovechar la diversidad \\
\hline Autorregulación & $\begin{array}{l}\text { - Orientación hacia el servicio } \\
\text { - Conciencia política }\end{array}$ \\
\hline - Escrupulosidad & \\
\hline $\begin{array}{l}\text { - Adaptabilidad } \\
\text { - Innovación }\end{array}$ & Habilidades sociales \\
\hline - Autocontrol & - Manejo de conflictos \\
\hline - Confiabilidad & - Catalizador de cambio \\
\hline Motivación & $\begin{array}{l}\text { - Colaboración y coperación } \\
\text { - Habilidades de equipo }\end{array}$ \\
\hline - Afán de triunfo & - Influencia \\
\hline - Compromiso & - Comunicación \\
\hline - Iniciativa & - Liderazgo \\
\hline - Optimismo & \\
\hline
\end{tabular}

Fuente: Elaboración propia incluyendo información de Goleman (2012)

Se considera que una persona puede desarrollar diferentes aptitudes personales y sociales en distinto grado, por lo que puede haber individuos con una inteligencia de la pasión desarrollada que difiere en el conjunto de las aptitudes adquiridas. El común denominador de la inteligencia de la pasión es la orientación de la atención y del esfuerzo al logro de objetivos.

\section{La falta de empatía en la inteligencia de la pasión}

A diferencia de la inteligencia emocional - que es moral y busca dirigir los pensamientos y las acciones de manera adecuada-, la inteligencia de la pasión no necesariamente es moral ni se limita al servicio de los demás. Esto no quiere decir que las personas que tengan desarrollada la inteligencia de la pasión sean inmorales o no tengan la capacidad de sentir empatía. Sin embargo, pueden o no tener desarrolladas las aptitudes relacionadas con la empatía y la moral.

Por tanto, se puede concebir a la inteligencia de la pasión como una inteligencia emocional incompleta al permitir que puedan estar ausentes aptitudes relacionadas con la empatía y las habilidades sociales. Para Vílchez (2018) "una deficiente inteligencia emocional o un mal uso de esta refuerzan o contribuyen a la desconexión moral” (p. 1). De hecho, "la desconexión moral sistémica ocurre cuando afecta a toda una sociedad o a parte de ella, que disculpa lo inmoral para justificar determinados comportamientos” (p. 2). En ese sentido, Gardner (2012) expone que "se debe aceptar la cruda realidad de que se puede ser 


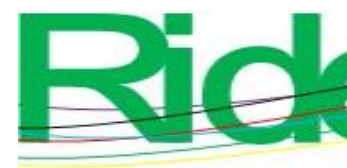

Revista Iberoamericana para la
Investigación y el Desarrollo Educativo
ISSN $2007-7467$

antisocial se refiere a "un grupo de conductas delictivas y antisociales; y a la psicopatía al conjunto de rasgos de la personalidad y conductas socialmente desviadas" (p. 45). Al respecto, Hare (2016) agrega que los psicópatas carecen completamente de conciencia y sentimientos por los demás. "Tienen una profunda incapacidad para preocuparse por el dolor y el sufrimiento ajeno, es decir, una falta total de empatía" (p. 24). Por otra parte, Robinett (2016) afirma que los psicópatas pueden ser “extremadamente atractivos y expertos en manipulación. Justifican sus opciones racionalmente, pero actúan estrictamente según sus intereses personales" (p. 76).

La empatía es uno de los componentes más importantes en las relaciones interpersonales. Es el vínculo entre la inteligencia emocional, la inteligencia social y la conciencia. Se trata de entender "cómo se sienten y piensan los demás, se relaciona con las habilidades sociales como la cooperación y el trabajo en equipo; y su fundamento neurocientífico forma parte de la neurociencia social" (Goleman y Senge, 2016, p. 33). La neurociencia social es una disciplina que estudia los circuitos cerebrales implicados en las interacciones. "Los circuitos sociales de la conciencia de uno mismo y de la autogestión se desarrollan en la infancia y son los que crean los instrumentos de la empatía y las habilidades sociales" (Goleman y Senge, 2016, p. 34).

Para Goleman (2015) existen tres clases de empatía: empatía cognitiva, empatía emocional y preocupación empática. La empatía cognitiva se refiere a ser capaz de percibir “cómo piensa otra persona; la empatía emocional a sentir en nosotros mismos las emociones de otra persona; y la preocupación empática a la sensibilidad hacia las necesidades de otra persona y la disposición de ayudar si es preciso" (pp. 159-160).

\section{La atención como núcleo central de la inteligencia de la pasión}

La atención es una destreza mental fundamental para el aprendizaje. "Según James la atención depende de un acto de voluntad que lleva a decidir qué es lo que se debe admitir en la mente mientras que para Freud depende del inconsciente, un dominio que las decisiones conscientes no pueden alcanzar" (Goleman, 2019, pp. 31-32). En ese sentido, el control cognitivo refiere a la capacidad para mantener la atención en un objetivo y al grado de atención que se presta para lograrlo. Según Goleman y Senge (2016), en un estudio realizado en la Universidad de Stanford se encontró que "el control cognitivo es un predictor de éxito en la vida. Influye en el futuro académico, en la salud y en la situación económica más que 


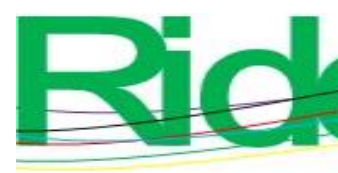

Revista Iberoamericana para la
Investigación y el Desarrollo Educativo
ISSN $2007-7467$

el CI y el estatus social y económico de la familia" (p. 27). Los autores añaden que "el cerebro utiliza los mismos circuitos para centrarse en un objetivo que para controlar emociones destructivas" (p. 25).

La concentración es la "capacidad neuronal de dirigir la atención hacia un solo objetivo ignorando lo demás, la atención obliga a desconectar las distracciones emocionales" (Goleman, 2013, p. 27), por lo que "apunta a fortalecer la habilidad mental de mantener la atención sin distraerse y centrarla en un objetivo" (Bennett-Goleman, 2019, p. 63). En ese sentido, Csikszentmihalyi (2000) agrega que "la búsqueda de un objetivo trae orden a la conciencia porque las personas deben concentrar su atención en la tarea que están llevando a cabo y olvidarse de todo lo demás" (p. 17). Goleman (2013) asegura que la concentración influye en el bienestar. La concentración aporta paz, alegría y permite a las personas tener pensamientos profundos y "mantener el rumbo en medio de una turbulencia emocional" (pp. 27). Goleman y Senge (2016) afirman que "la atención se puede mejorar y fomentar" (p. 28) con entrenamiento, ya que es una capacidad limitada (Bennett-Goleman, 2019).

\section{El entrenamiento de la mente para la atención: meditación}

Entrenar la atención puede reducir la ansiedad y las distracciones. De acuerdo con Bennett-Goleman (2019), se puede entrenar la atención mediante la meditación desde dos enfoques: concentración y percepción. La meditación “es una herramienta que permite explorar y trabajar el mundo interno y manejar los pensamientos hacia un objetivo" (Raij, 2019, p. 146). Gordon, Posner, Klein y Mumm (2019) afirman que la meditación mejora la concentración, la memoria y puede convertirte en maestro de la mente.

Por su parte, Puddicombe, Rogoway y Selzer (2021) argumentan que la meditación es la habilidad para entrenar la mente de manera que podamos disfrutar más la vida. Estos autores aseguran que en investigaciones realizadas en la Universidad Harvard se encontró que, a partir de la meditación durante ocho semanas consecutivas, el cerebro experimenta cambios físicos y psicológicos: las partes relacionadas con el bienestar y la felicidad reciben más sangre y físicamente se hacen más grandes, mientras que las partes donde reside el estrés y la ansiedad se reducen. En ese sentido, la Universidad de Wisconsin desarrolló un videojuego que llamó tenacidad para promover la concentración y entrenar la atención (Goleman y Senge, 2016, p. 29); y así como también se han desarrollado aplicaciones móviles electrónicas como headspace y calm que promueven la meditación (Gordon et al., 2019). 


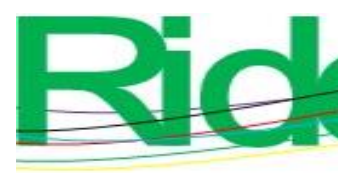

Revista Iberoamericana para la
Investigación y el Desarrollo Educativo
ISSN $2007-7467$

consecutivas de realizar ejercicios de meditación los alumnos mejoraron la manera en que se relacionaban entre ellos.

\section{La pasión en la educación}

La pasión — según Ramos y González (2020)— debe considerarse como parte importante en el proceso de orientación vocacional debido a la relevancia que tiene en el sentido de vida de los estudiantes. Urbina y Ávila (2013) aseguran que en el aprendizaje las pasiones "son como motores que mueven a los estudiantes a aprender" (p. 3). Además, definen la pasión por aprender como un "acto natural y permanente donde se comprometen todos los sentidos para lograr saciar el infinito afán de saber" (p. 4). La pasión por aprender implica razón, emoción, reto, esfuerzo, dedicación y responsabilidad, por lo que Ubina y Ávila (2013) la denominan pasión inteligente. "Una pasión inteligente es una aleación de sabiduría y de previsión científica, que actúa en el momento de aprender como la forma singular de vivir de un ser humano, a manera de acontecimiento" (Urbina y Ávila, 2013, p. 7).

La pasión inteligente surge de manera natural cuando corresponde a "un gusto o interés personal, a la voluntad propia del sujeto y a su vocación individual” (Urbina y Ávila, 2013, p. 12). En contraste, cuando las tareas son obligadas y van en contra de la vocación o intereses, la pasión se diluye. De tal manera que la vocación juega un papel fundamental que impacta en el rendimiento académico de los estudiantes y en su desempeño laboral futuro. En un estudio realizado sobre satisfacción vocacional en universitarios se encontró que $62 \%$ de los egresados no estaban satisfechos con la carrera elegida. El factor más relevante fue el “desencanto con la profesión”, lo cual sucede porque en muchos casos las personas se ven “obligadas a estudiar sin vocación alguna” (Zilvetty, 2019, p. 8).

Para Urbina y Ávila (2013) "no hay aprendizaje significativo sin pasión, y no hay pasión sin encontrar significado a lo aprendido" (p. 7). Por otra parte, Fried (1995, citado por Day, 2006) asegura que "la pasión puede descubrirse, enseñarse o reproducirse y está en el centro de la enseñanza" (p. 27). En ese sentido, para lograr un aprendizaje significativo en los estudiantes se debe considerar la importancia que tiene la alineación de sus intereses y su vocación con las tareas y actividades requeridas. Esto significa que la pasión por aprender se deriva de una motivación intrínseca por el conocimiento que, al mismo tiempo, genera placer. Para conseguir este propósito, los docentes deben ser capacitados en competencias sociales 


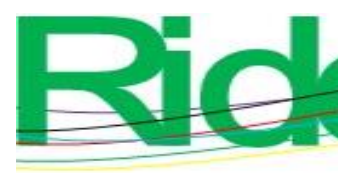

Revista Iberoamericana para la
Investigación y el Desarrollo Educativo
ISSN $2007-7467$

y neurociencia para comprender los cambios y las reacciones que se producen en el cerebro frente a las emociones (Jiménez et al., 2020).

Por otra parte, Gardner (2012) explica que la educación para ser eficaz debe incluir en la práctica diaria "la motivación, las emociones, valores sociales y morales" (p. 24). Asimismo, considera que las sociedades para ser competitivas deben ofrecer a la mayoría de sus ciudadanos una educación que "responda con agilidad a seis conjuntos de tendencias: descubrimientos tecnológicos y científicos; tendencias políticas, fuerzas económicas; tendencias sociales, culturales y personales en la era moderna; la cambiante cartografía del conocimiento y al punto de vista multicultural" (p. 48).

Gardner (2012) opina que "cualquier descripción de la naturaleza humana que ignore la motivación y la emoción tiene una utilidad limitada para facilitar el aprendizaje y la pedagogía". Por ende, asegura que "para que los estudiantes aprendan, dominen y apliquen algo con criterio, se necesita envolver ese algo en un contexto que haga intervenir las emociones" (p. 89). Por otra parte, Cerda (2000, citado por Navarro, 2020) afirma que "la habilidad del cerebro para mostrar curiosidad por lo novedoso facilita el aprendizaje y el pensamiento creativo complementa al pensamiento analítico para resolver problemas” (p. 7). Por tanto, se resalta la necesidad de escuelas que promuevan entornos y ambientes positivos, que favorezcan la curiosidad de los estudiantes, el disfrute, el estímulo y el desafío.

En ese sentido, la educación moral con convicción e inteligencia emocional propuesta por Vílchez (2018) que busca enseñar a los niños y jóvenes a ser verdaderos ciudadanos recorriendo el camino de la esperanza y de la paz puede fungir como estrategia para lograr el nuevo modelo de humanidad señalado por Gardner (2012), donde las personas "además de saber analizar, también busquen hacer lo correcto; personas que no solo sean admirables como creadoras sino también como seres humanos" (p. 283). 


\section{Conclusiones}

La educación integral se puede entender como un rompecabezas donde las ciencias, las disciplinas y los diferentes paradigmas psicopedagógicos se convierten en piezas clave para lograr el objetivo fundamental de la educación: formar personas y exponer lo verdadero, lo bello y lo bueno. A lo largo de la historia, la educación se ha transformado de tal manera que surgen nuevas disciplinas y líneas de investigación con el objetivo de profundizar y crear nuevos conocimientos que permitan comprender al ser humano y formarlo para que contribuya a mejorar la sociedad a la que pertenece.

En este sentido, en el presente trabajo se ha concebido a la pasión como una fuerza motivadora intrínseca insaciable y permanente que provoca satisfacción al tratar de conseguir el objeto deseado. La característica insaciable de la pasión es lo que permite que tenga duración a lo largo del tiempo y la hace permanente, pues nunca se obtiene suficiente del objeto deseado, lo que crea una dependencia del sujeto para el objeto. Sin embargo, lo que para antiguos filósofos como Kant resulta negativo de la pasión (las características de la dependencia y el apetito insaciable), ahora se le da un propósito, pues la pasión se puede orientar hacia el logro de objetivos. En otras palabras, la dependencia y el apetito insaciable de la pasión brindan propósito, convicción y dirección al permitir que el sujeto que la posee domine y perfeccione técnicas, estrategias, disciplinas, oficios, etc. La pasión, entonces, abre la puerta al origen de pasiones inteligentes, como la pasión por enseñar, por aprender, por emprender, por la ciencia o por el trabajo, lo que también implica esfuerzo, dedicación, concentración y orden. Las pasiones inteligentes ayudan al sujeto a lograr sus objetivos al mismo tiempo que sienten una satisfacción durante el proceso.

En este contexto se introduce la inteligencia de la pasión como la capacidad para lograr objetivos y resolver problemas de diferente índole a través del enfoque de la atención a los resultados deseados. Al ser la atención la base fundamental de este tipo de inteligencia, esta puede desarrollarse mediante el entrenamiento de la mente para mejorarla. La meditación es una estrategia para entrenar a la mente, mejorar la atención y la concentración. La meditación constante produce beneficios para la salud física, emocional y mental, y permite relacionarse con el ambiente y con las demás personas de una manera más armoniosa, por lo que facilita una convivencia positiva y sana.

Por otra parte, vale destacar que el aprendizaje y las maneras de transmitir conocimiento también se pueden auxiliar de la tecnología. Aplicaciones tecnológicas móviles 


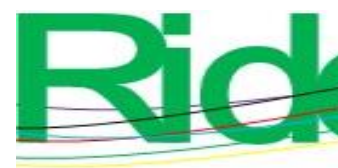

Revista Iberoamericana para la Investigación y el Desarrollo Educativo ISSN 2007-7467

como Headspace y Calm permiten a las personas comenzar a explorar la meditación, la cual se sugiere incorporar como parte de las estrategias institucionales en el sector educativo con el objetivo de brindar a los jóvenes herramientas y técnicas que les permitan focalizar su atención en el logro de objetivos al mismo tiempo que incrementan su bienestar físico y psicológico.

Para ello, por supuesto, se necesitan docentes que sean capaces de despertar la pasión por aprender en los estudiantes, los cuales cuando se encuentren en el mercado laboral podrán ser más apasionados, productivos y disfrutar de su profesión.

\section{Referencias}

Bennett-Goleman, T. (2019). Alquimia emocional. España: Penguin Random House.

Burlando, G. (2011). Meditaciones morales de Descartes: pasión y auto conservación de la vida. Veritas, (25), 75-91.

Cantón, E., Sánchez, A. y Peris, D. (2019). Intervención clínica desde la psicología motivacional en una gimnasta joven de alta competición. Horizonte Sanitario, 18(3).

Centeno, M. (2019). Arregla tu vida con grafologia. México: Aguilar.

Csikszentmihalyi, M. (2000). Fluir. España: Kairós.

Day, C. (2006). Pasión por enseñar. Madrid: Narcea.

Ferreiro, R. (2012). La pieza clave del rompecabezas del desarrollo de la creatividad: La escuela. Revista Iberoamericana sobre Calidad, Eficacia y Cambio en Educación, $10(2), 6-22$.

García, D., Álvarez, E., González, E. y Guzmán, D. (2019). La nueva Licenciatura en Neurociencias de la UNAM: lecciones aprendidas. Investigación en Educación Médica, 8(29), 104-109. Doi: https://doi.org/10.22201/facmed.20075057e.2019.29.18104

Gardner, H. (2012). La educación de la mente y el conocimiento de las disciplinas. España: Paidós.

Goleman, D. (1995). Inteligencia emocional. México: Ediciones B.

Goleman, D. (2006). Inteligencia social. España: Kairós.

Goleman, D. (2012). La inteligencia emocional en la empresa. Argentina: Ediciones B.

Goleman, D. (2013). Focus: desarrollar la atención para alcanzar la excelencia. México: Kairós. 


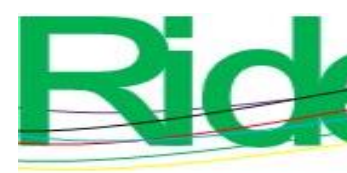

Revista Iberoamericana para la Investigación y el Desarrollo Educativo ISSN $2007-7467$

Goleman, D. (2015). Como ser un líder. España: Ediciones B.

Goleman, D. (2019). El punto ciego. México: Penguin Random House.

Goleman, D. y Senge, P. (2016). Triple focus. México: Ediciones B.

Goleman, D., Kaufman, P. y Ray, M. (2016). El espíritu creativo. México: Ediciones B.

González, A. (2015). Emoción, sentimiento y pasión en Kant. Trans-Form-Açao: Revista de Filosofía, 38(3), 75-98. Doi: http://dx.doi.org/10.1590/S0101-31732015000300006

Gordon, C., Posner, J., Klein, E. y Mumm, C. (productores) (2019). La mente, en pocas palabras [documental]. Estados Unidos: Netflix.

Hare, R. (2016). Sin conciencia. México: Paidós.

Holley, K. (2009). The challenge of an interdisciplinary curriculum: A cultural analysis of a doctoral-degree program in neuroscience. Higher Education, 58(2), 241-55. Doi: https://doi.org/10.1007/s10734-008-9193-6

Jiménez, J., Mendoza, P., Aimaretti, J., Flores, P., Montalvo, M. y Mena, O. (2020). Proceso de rendimiento académico con la identidad universitaria en argentinos, peruanos, mexicanos y costarricenses considerando factores de la neurociencia. RIDE Revista Iberoamericana para la Investigación y el Desarrollo Educativo, 10(20). Doi: https://doi.org/10.23913/ride.v10i20.597

Lázaro, R. (2012). Descartes y las pasiones del alma. Cauriensia, 7, 249-257.

Márquez, M., Azofeita, C. y Rodríguez, C. (2019). Factores de motivación de logro: el compromiso y entrega en el aprendizaje, la competencia motriz percibida, la ansiedad ante el error y situaciones de estrés en estudiantes de cuarto, quinto y sexto nivel escolar durante la clase de educación física. Revista Educación, 43(1). Doi: http://dx.doi.org/10.15517/revedu.v43i1.33109

Mora, J. y Martin, M. (2007). La concepción de la inteligencia en los planteamientos de Gardner (1983) y Sternberg (1985) como desarrollos teóricos precursores de la noción de inteligencia emocional. Revista de Historia de la Psicología, 28(4), 67-92.

Navarro, M. (2020). La creatividad en la formación del arquitecto, el proceso creativo y las neurociencias. RIDE Revista Iberoamericana para la Investigación y el Desarrollo Educativo, 10(20). Doi: https://doi.org/10.23913/ride.v10i20.667

Nelson, B. (2019). The emotion code. New York: St. Martin’s Essentials.

Pimienta, J., De la Orden, A. y Estrada, R. (2018). Metodología de la investigación. México: Pearson Educación de México. 


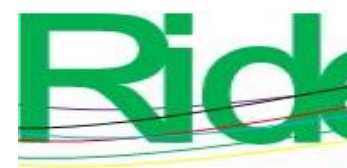
Revista Iberoamericana para la
Investigación y el Desarrollo Educativo
ISSN $2007-7467$

Puddicombe, A., Rogoway, S. y Selzer, M. (productores ejecutivos). (2021). Guía headspace para la meditación [serie de televisión]. Estados Unidos: Netflix.

Raij, S. (2019). Full Stop. México: Paidós.

Ramírez, J. (2010). Corrientes filosóficas que sustentan a la educación física, el deporte y la recreación. Recorde: Revista de História do Esporte, 3(1).

Ramos, C. y González, B. (2020). Orientación vocacional, aprendizaje socioemocional y sentido de vida en la educación superior. Revista Dilemas Contemporáneos: Educación, Política y Valores, 8(21). Doi: https://doi.org/10.46377/dilemas.v8i.2500

Robinett, J. (2016). Networking estratégico. México: Paidós.

Sánchez, R., Costa, O., Mañoso, L., Novillo, M. y Pericacho, F. (2019). Orígenes del conectivismo como nuevo paradigma del aprendizaje en la era digital. Revista Educación y Humanismo, 21(36), 113-136.

Secretaría de Educación de Tamaulipas (SET). (2017). Modelo educativo capítulo Tamaulipas 2016-2022. Recuperado de www.tamaulipas.gob.mx/educacion/modeloeducativo-capitulo-tamaulipas/

Selles, J. (2007). Las dualidades de la educación. Educación y Educadores, 10(1), 135-160.

Tacca, D., Tacca, A. y Alva, M. (2019). Estrategias neurodidácticas, satisfacción y rendimiento académico en estudiantes universitarios. Cuadernos de Investigación Educativa, 10(2), 15-32. Doi: https://doi.org/10.18861/cied.2019.10.2.2905

Trujillo, S. (2007). Objetividad y sujetualidad: una perspectiva del debate epistemológico en psicología. Tesis Psicológica, (2), 75-79.

Universidad Autónoma de Tamaulipas (UAT) (2021). Lema de la UAT. Recuperado de www.uat.edu.mx/paginas/universidad/identidad.aspx

Urbina, J. y Ávila, R. (2013). Sentidos de la pasión de aprender. Perspectiva de estudiantes de universidad. Revista Latinoamericana de Ciencias Sociales, Niñez y Juventud, 11(2), 803-817.

Vílchez, L. (2018). "Participación educativa con inteligencia emocional y moral". Participación Educativa, 5(8), 43-54.

Zilvetty, M. (2019). Déficit de titulación y su relación con la satisfacción vocacional y profesional. Educación Superior, 6(1), 52-59. 